\title{
Primary Melanocytic Lesion of Meninges
}

National Cancer Institute

\section{Source}

National Cancer Institute. Primary Melanocytic Lesion of Meninges. NCI Thesaurus. Code C4661.

A benign or malignant, circumscribed or diffuse neoplasm that arises from melanocytes in the leptomeninges. It includes diffuse melanocytosis, leptomeningeal melanoma, and melanocytoma. 